\title{
Modelo heurístico híbrido para el ruteo vehicular y manejo de inventario en una entidad comercializadora de combustibles
}

\author{
FRÓMETA MOYA, JORGE ISRAEL \\ Universidad de Oriente, Santiago de Cuba (Cuba) \\ Correo electrónico: jorgef@uo.edu.cu \\ PÉREZ CAMPos, JAVIER DE JESÚS \\ Universidad de Oriente, Santiago de Cuba (Cuba) \\ Correo electrónico: jperez@refscu.cupet.cu
}

\begin{abstract}
RESUMEN
El problema de ruteo vehicular combinado con el manejo de inventario es un tema complejo reconocido en su tratamiento por la literatura científica. En este trabajo se presenta un problema de este tipo con la particularidad que tiene como objetivo la optimización del indicador tráfico de carga, así como un modelo y procedimiento matemático para la resolución de este problema en una entidad comercializadora de combustibles de la región oriental de Cuba. Para ello se utilizaron métodos heurísticos combinados, de descomposición y reducción, integrados con programación lineal binaria, para la determinación del ruteo vehicular hacia los clientes. Todo ello para simplificar el problema y ofrecer una solución satisfactoria basada en el cumplimiento de niveles de inventarios de acuerdo a un nivel de cobertura planificado y el uso racional de los vehículos. Finalmente, se probó en un caso de estudio basado en una situación real operativa de la empresa en cuestión.
\end{abstract}

Palabras clave: ruteo vehicular, manejo de inventarios, métodos heurísticos, programación lineal binaria.

Clasificación JEL: C61.

MSC2010: 90Bxx 


\title{
Hybrid heuristic model for inventory routing management in a fuel comercial entity
}

\begin{abstract}
The inventory routing problem is a complex topic recognized by the scientific literature. This work shows up this kind of problem, particularly a problem with freight traffic indicator as an objective for optimization, also a mathematical model and procedure for the resolution of this problem in an entity that sales fuel in eastern region of Cuba. For it, we were used heuristics combined methods of decomposition and reduction, integrated with binary linear programming, for the determination of the routing for vehicles toward the clients. The objective was to simplify the problem and to offer a satisfying performance based on the achievement of inventory levels according to a planned covering level, the rational use of the vehicles. Finally, it was proven in a case of study based on a real operative situation of the company studied.
\end{abstract}

Keywords: vehicle routing problem, inventory management, heuristics methods, binary linear programming.

JEL classification: C61.

MSC2010: 90Bxx

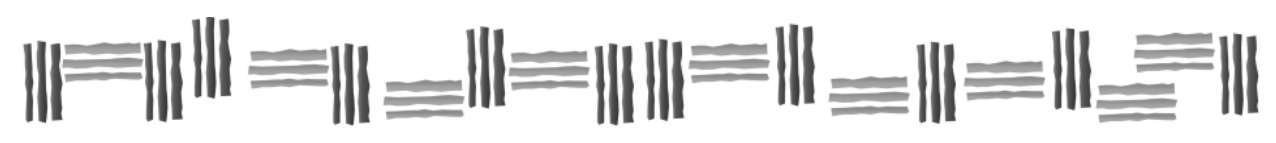




\section{Introducción.}

El ámbito empresarial del siglo XXI exige a las empresas explotar continuamente oportunidades de mejora en sus procesos a fin de ser competitivas y responder con eficiencia y eficacia las necesidades y requerimientos de sus clientes. Es en este contexto donde la logística y sus subsistemas asociados, tales como aprovisionamiento, distribución, inventarios, etc., cobra especial importancia y por ello numerosas investigaciones se orientan a la mejora del desempeño en estos aspectos. Como herramienta invaluable en la toma de decisiones logísticas se encuentra la modelación matemática enmarcada en la Investigación de Operaciones (Lao, Vega, Marrero \& Pérez, 2017). Dentro de ésta, la literatura ha prestado especial atención al problema de ruteo vehicular (VRP por sus siglas en inglés) como nombre genérico dado a la clase de problemas de naturaleza combinatoria donde se busca optimizar el rendimiento de una flota de vehículos, cuando éstos ejecutan sus viajes como parte de un itinerario (Cattaruzza, Absi, Feillet \& González-Feliu, 2017).

De acuerdo a Eksioglu, Vural y Reisman (2009), la literatura sobre VRP ha ido creciendo a un ritmo de 6\% por año (Braekers, Ramaekers \& Van Nieuwenhuyse, 2016). Es por ello que existe una infinidad de variantes o extensiones del VRP dependiendo de las variables, objetivos, restricciones y dimensiones a considerar, entre las que se pueden destacar: el Traveling Salesman Problem (TSP) (Palhares \& Araújo, 2018), Capacitated Vehicle Routing Problem (CVRP) (Uchoa et al., 2017), Vehicle Routing Problem with Time Windows (VRPTW) (Pecin et al., 2017), Dynamic Vehicle Routing Problem (Ritzinger, Puchinger \& Hartl, 2016), Inventory Routing Problem (IRP) (Coelho, Cordeau \& Laporte, 2013), entre muchos otros. El problema del ruteo vehicular asociado al manejo de inventario, o Inventory Routing Problem (IRP), constituye una variante que se puede presentar cuando en un sistema logístico el distribuidor y sus clientes se relacionan bajo el principio Vendor Managed Inventory (VMI) en los que el distribuidor toma control de los inventarios de cada uno, asegurándole que se mantendrán niveles adecuados de inventarios (Campbell et al., 1998; Coelho et al., 2014).

En este trabajo se propone un modelo y procedimiento matemático heurístico que integra la planificación del transporte y el manejo de inventario como solución a un problema IRP, dado en el contexto de la planificación operativa de una empresa de distribución de combustibles. El problema que se pretende solucionar con la propuesta involucra la optimización del indicador tráfico de carga como función de valor del rendimiento de la flota de vehículos, así como el mantenimiento de los inventarios en los niveles planificados. En la concepción del modelo se emplean métodos heurísticos de descomposición y reducción, combinados con la programación lineal binaria como método de optimización exacto con un enfoque monobjetivo.

Las contribuciones principales de este trabajo son: (1) introducir un nuevo modelo y procedimiento matemático como solución de un problema de ruteo vehicular asociado al manejo de inventario en una entidad dedicada a la distribución de combustibles, cuyas particularidades en el indicador y objetivo que se persigue no se encuentra recogido en la literatura revisada; y (2) aplicar el modelo propuesto a una situación real de la planificación operativa de la empresa en cuestión y comparar los resultados respecto a los resultados obtenidos sin la aplicación del modelo.

El artículo se estructura de la siguiente forma. En la Sección 2 se presenta una revisión de la literatura relacionada con el problema IRP y las clasificaciones propuestas para el mismo. En la Sección 3 se propone el modelo matemático asociado al problema, el procedimiento de resolución y los diferentes métodos heurísticos y de optimización empleados. A continuación, en la Sección 4 se describe el caso de estudio, evaluándose los resultados obtenidos por la aplicación del modelo y el procedimiento matemático propuesto respecto a la planificación actual. Por último, se presentan las conclusiones y las líneas futuras de investigación. 


\section{Revisión de la literatura.}

En esta sección se presenta la revisión de la literatura que fundamenta el vacío que se presenta en ésta respecto a la principal contribución de este trabajo. Existe una cantidad significativa de trabajos científicos publicados respecto al problema IRP. Una referencia abarcadora e interesante sobre el tema sería Coelho et al. (2014). La búsqueda bibliográfica se enfoca en los siguientes aspectos: clasificación del problema IRP, así como objetivos e indicadores claves que lo representa.

La compleja tarea de desarrollar una estrategia de distribución que optimice los niveles de inventario y al mismo tiempo optimice el rendimiento del proceso de transporte es lo que la literatura recoge como el problema del ruteo vehicular asociado al manejo de inventario, o Inventory Routing Problem (IRP). El IRP difiere del tradicional problema de ruteo vehicular (VRP), en que está basado en los niveles de consumo e inventario de los clientes y no en las órdenes de reaprovisionamiento de éstos (Campbell et al., 1998).

El problema IRP por su complejidad cuenta con diferentes variantes, dependiendo de parámetros como el horizonte de tiempo a planificar, o el número de viajes permitidos de cada vehículo, o las características del inventario y la demanda que genera (Campbell et al., 1998). Soysal, BloemhofRuwaard, Haijema y Van der Vorst (2018) presentan una clasificación basada en el número de suministradores y clientes en la relación que se establece como parte del problema. No obstante, la mayoría de las investigaciones sobre este problema se pueden focalizar en tres variantes (Campbell \& Savelsbergh, 2004):

- Modelo de planificación de un solo día con demanda estocástica o determinística.

- Modelo de planificación para varios días con demanda estocástica o determinística.

- Modelo de planificación permanente, con el propósito de una planificación a largo plazo.

Rahim, Iteng y Ahmad (2017) muestran una clasificación un poco más explícita del problema IRP definiendo una subclase dentro de este tipo de problema que denomina single-period deterministic inventory routing problem (SP-DIRP) donde la demanda de los clientes en un periodo de tiempo es asumida como determinada. Una clasificación similar es dada por autores como Andersson, Hoff, Christiansen, Hasle y Løkketangen (2010), así como Li, Chen, Sivakumar y Wu (2014). El problema en que se enfoca este trabajo, con determinadas particularidades, puede enmarcarse en la clasificación dada por estos autores.

Por otra parte, las diferentes investigaciones sobre IRP también tienen múltiples enfoques en lo referente a los objetivos que se persiguen con la solución de este tipo de problemas. Archetti, Coelho y Speranza (2019) hacen un análisis de diferentes estudios sobre IRP basado en las funciones objetivos que se proponen en los mismos, clasificándolos esencialmente en mono objetivos y multiobjetivos. El problema IRP clásico se enfoca en minimizar el costo total, combinando los costos del ruteo y el inventario con un enfoque mono objetivo. Recientemente, otros objetivos han sido propuestos con la finalidad de mejorar el desempeño de la cadena logística o para adaptar soluciones específicas (Archetti et al., 2019). Entre estos otros objetivos se pueden enunciar la minimización de las emisiones contaminantes y los desperdicios (Soysal et al., 2018).

Una interesante alternativa es la optimización de la razón logística o "logistic ratio", propuesta por Archetti, Desaulniers y Speranza (2017), que consiste en la razón entre el costo de la distribución y la cantidad total distribuida, siendo el costo promedio de distribuir cada unidad con la finalidad de optimizar el impacto en la planeación a corto y largo plazo. No obstante, no se encontró en la literatura científica revisada referente al problema IRP en su definición más global, ni en la que se refiere al caso específico del Single-Period Deterministic Inventory Routing Problem (SP-DIRP), ninguna propuesta cuya función objetivo incluyera la optimización del indicador tráfico de carga, que es "la magnitud del trabajo en el transporte de carga combinando la carga y la distancia a la que se transportan los productos 
y mercancías" (ONEI, 2019). La utilización de este indicador como expresión del objetivo a optimizar representa la principal peculiaridad del problema en que se enfoca el presente trabajo.

\section{Formulación del modelo.}

\subsection{Definición del problema.}

El problema considerado en este trabajo es del tipo Single-Period Deterministic Inventory Routing Problem (SP-DIRP), teniendo como como criterio de valor de las soluciones el indicador tráfico de carga. La propuesta que se propone persigue superar insuficiencias en el proceso de distribución y manejo de inventario en una empresa de distribución de combustibles en Cuba, que afectan la eficiencia y la eficacia que demanda dicho proceso.

Se definieron los supuestos que sirven de base para el modelo económico matemático:

- Se quiere satisfacer la demanda lo más rápido posible y al menor costo priorizando a partir del nivel de cobertura planificado y sin excederse de éste.

- Existe un único tipo de mercancías a transportar.

- Cada camión transporta el valor de toda su carga o el valor de la demanda del destino en cada visita.

- La flota de vehículos es limitada, heterogénea, con restricciones de capacidad pero todos estos aspectos son determinados.

- Las distancias entre los puntos físicos del modelo son conocidas; la velocidad de los vehículos y los costos asociados son proporcionales a las distancias recorridas, aunque estos valores son indeterminados e independientes del volumen de carga transportada y del tipo de vehículo o el destino.

- Cada vehículo solo carga en un único punto de origen, no existe trasbordo.

- Cada destino tiene un inventario de existencias de mercancías conocido y un consumo promedio diario también conocido de estas mercancías.

- Se utiliza un horizonte de tiempo diario para la programación del ruteo vehicular; fuera de esto, no existe ventana u horario de tiempo limitante.

- No se permiten transportes simultáneos de vehículos a un mismo destino.

\subsection{Formulación del modelo matemático.}

El modelo matemático propuesto, se encuentra estructurado por etapas, a partir de la aplicación de un método heurístico de descomposición:

Definición de Etapas (n): identificación ordinal de la secuencia de visitas en orden cronológico que realiza la flota de vehículos de manera simultánea a los destinos.

$$
\mathrm{n}=\{1,2, \ldots, \mathrm{N}\} \text {, siendo } \mathrm{N} \text { el número total de etapas, } \mathrm{n} \in \mathbb{N} \text {; }
$$

Definición de los parámetros del modelo: mediante estos parámetros se reflejan las condiciones del problema independientemente de la etapa en que se encuentre o de las decisiones que se adopten. 


\section{En el caso de los vehículos:}

I: Conjunto que identifica la flota de vehículos disponibles para el transporte de mercancías hacia los destinos, este conjunto estará conformado por los elementos i donde:

i: Identificación única de cada vehículo:

$\mathrm{i}=\{1,2, \ldots, \mathrm{m}\} / \mathrm{m}$ es el número total de vehículos disponibles, $\mathrm{i} \in \mathrm{I}$;

Cada vehículo tiene asociado un parámetro determinado por:

$\mathrm{C}_{\mathrm{i}}$ : Carga del volumen de mercancías del vehículo i cuando visita el origen, tal que:

$\mathrm{i} \rightarrow \mathrm{C}_{\mathrm{i}} / \mathrm{C}_{\mathrm{i}} \in \mathbb{R}, \mathrm{C}_{\mathrm{i}}>0$;

\section{En el caso de los destinos:}

J: Conjunto que identifica los destinos a los que se puede abastecer de mercancías. Este conjunto estará conformados por los elementos $\mathrm{j}$ donde:

j: Identificación única de cada destino:

$\mathrm{j}=\{1,2, \ldots, \mathrm{L}\} / \mathrm{L}$ es el número total de destinos, $\mathrm{j} \in \mathrm{J}$;

Cada destino j tiene asociado un vector de parámetros de dimensión $\mathbb{R}^{4}$ :

$\mathrm{j} \rightarrow\left(\mathrm{T}_{\mathrm{j}}, \mathrm{p}_{\mathrm{j}}, \mathrm{b}, \mathrm{E}_{\mathrm{j}}\right)$

$\mathrm{T}_{\mathrm{j}}$ : Inventario de mercancías en el destino $\mathrm{j}$ al inicio del problema.

$\mathrm{p}_{\mathrm{j}}$ : Posición física del destino $\mathrm{j}$.

b: Tiempo de cobertura planificado para cualquier destino j para todo el problema.

$\mathrm{E}_{\mathrm{j}}$ : Consumo de mercancías promedio de cada destino $\mathrm{j}$.

\subsection{Parámetros auxiliares.}

$\mathrm{R}_{\left(\mathrm{p}_{1}, \mathrm{p}_{2}\right)}$ : Distancia existente entre un punto físico 1 del modelo hasta otro punto 2 .

Definición de variables de estado en cada Etapa del modelo: refleja la condición o estado del modelo que enlazan las etapas. Representa la relación entre etapas de tal manera que cuando cada etapa se optimiza por separado, la decisión resultante es automáticamente factible para el problema completo.

\section{En el caso de los vehículos:}

El conjunto I tiene asociados $N$ conjuntos $I_{n}$, uno por cada etapa que se analiza, de modo que:

$\mathrm{I}_{\mathrm{n}}$ : Conjunto que identifica la flota de vehículos disponibles para el transporte de mercancías hacia los destinos en cada etapa $n$. Este conjunto estará conformado por los elementos $i_{n}$ donde:

$\mathrm{i}_{\mathrm{n}}$ : Identificación única de cada vehículo en cada etapa $\mathrm{n}$.

Cada vehículo tiene asociado en cada etapa un vector de variables de dimensión $\mathbb{R}^{2}$ : 
$\mathrm{i}_{\mathrm{n}} \rightarrow\left(\mathrm{c}_{\mathrm{i}_{\mathrm{n}}}, \mathrm{p}_{\mathrm{i}_{\mathrm{n}}}\right)$

$\mathrm{c}_{\mathrm{i}_{\mathrm{n}}}$ : Carga del volumen de mercancías del vehículo i al inicio de cada etapa $\mathrm{n}$.

$p_{i_{n}}:$ Posición física del vehículo i al inicio de cada etapa n:

$\mathrm{p}_{\mathrm{i}_{\mathrm{n}}}=\{0,1, \ldots, \mathrm{L}\} /$ siendo $1,2, \ldots$, hasta $\mathrm{L}$ los números que identifican la posición física de cada destino, $0=$ origen;

\section{En el caso de los destinos:}

El conjunto $\mathrm{J}$ tiene asociados $\mathrm{N}$ conjuntos $\mathrm{J}_{\mathrm{n}}$, uno por cada etapa que se analiza, de modo que:

$\mathrm{J}_{\mathrm{n}}$ : Conjunto que identifica los destinos en cada etapa $\mathrm{n}$ con demanda sin satisfacer al inicio de dicha etapa de acuerdo al nivel de cobertura planificado, este conjunto estará conformados por los elementos $j_{n}$ donde:

$\mathrm{J}_{\mathrm{n}} \subseteq \mathrm{J}, \forall \mathrm{n} ;$

$\mathrm{j}_{\mathrm{n}}$ : Identificación única de cada destino con demanda sin satisfacer de acuerdo al nivel de cobertura planificado en cada etapa $n$.

Cada destino $\mathrm{j}_{\mathrm{n}}$ tiene asociado al inicio de cada etapa un vector de variables de dimensión $\mathrm{R}^{4}$ :

$\mathrm{j}_{\mathrm{n}} \rightarrow\left(\mathrm{t}_{\mathrm{j}_{\mathrm{n}}}, \mathrm{w}_{\mathrm{j}_{\mathrm{n}}}, \mathrm{a}_{\mathrm{j}_{\mathrm{n}}}, \mathrm{d}_{\mathrm{j}_{\mathrm{n}}}\right)$

$\mathrm{t}_{\mathrm{j}_{\mathrm{n}}}$ : Inventario de mercancías en el destino $\mathrm{j}_{\mathrm{n}}$ al inicio cada etapa $\mathrm{n}$.

$\mathrm{w}_{\mathrm{j}_{\mathrm{n}}}$ : tiempo de cobertura del inventario en cada etapa $\mathrm{n}$ del destino $\mathrm{j}_{\mathrm{n}}$ con respecto a su demanda diaria promedio.

$\mathrm{a}_{\mathrm{j}_{\mathrm{n}}}$ : orden de prioridad en la atención de la demanda de cada destino $\mathrm{j}_{\mathrm{n}}$ de acuerdo a su tiempo de cobertura en la etapa $n$.

$\mathrm{d}_{\mathrm{j}_{\mathrm{n}}}$ : Demanda de mercancías del destino $\mathrm{j}_{\mathrm{n}}$ en la etapa $\mathrm{n}$ en dependencia de su inventario de mercancías, su demanda promedio diaria y el tiempo de cobertura planificado.

Cada conjunto $\mathrm{J}_{\mathrm{n}}$ tiene asociado en cada etapa un conjunto $\mathrm{J}_{\mathrm{n}}^{\mathrm{k}}$ definido por la aplicación de una heurística de reducción del espacio vectorial de soluciones factibles, de modo que:

$\mathrm{J}_{\mathrm{n}}^{\mathrm{k}}$ : Conjunto que identifica los destinos en cada etapa $\mathrm{n}$ con demanda sin satisfacer al inicio de dicha etapa de acuerdo al nivel de cobertura planificado siendo escogidos por la aplicación de la heurística de acuerdo a su nivel de prioridad. Este conjunto estará conformado por los elementos $\mathrm{j}_{\mathrm{n}}^{\mathrm{k}}$ donde:

$\mathrm{J}_{\mathrm{n}}^{\mathrm{k}} \subseteq \mathrm{J}_{\mathrm{n}}, \quad \forall \mathrm{n}$ y $\forall \mathrm{k} ;$

$\mathrm{j}_{\mathrm{n}}^{\mathrm{k}}$ : Identificación única de cada destino $\mathrm{j}$ en la etapa n que forma parte como elemento $\mathrm{k}$ del vector de destinos escogidos por la heurística de reducción del espacio vectorial de soluciones factibles en la etapa $n$. 


\section{Definición de los coeficientes de contribución de la función objetivo en cada etapa:}

$\mathrm{r}_{\left(\mathrm{p}_{\mathrm{i}_{\mathrm{n}}}, \mathrm{p}_{\mathrm{j}} \mathrm{k}\right.}$ : Distancia a recorrer por el vehículo i desde su posición al inicio de la etapa $\mathrm{n}$ hasta la posición de un destino $\mathrm{j}_{\mathrm{n}}^{\mathrm{k}}$.

$\mathrm{y}_{\left(\mathrm{i}_{\mathrm{n}}, \mathrm{j}_{\mathrm{n}}^{\mathrm{k}}\right)}$ : Cantidad de mercancías a transportar por el vehículo i al destino $\mathrm{j}_{\mathrm{n}}^{\mathrm{k}}$ en dependencia de la carga del vehículo y la demanda del destino en la etapa n.

$\mathrm{v}_{\left(\mathrm{i}_{\mathrm{n}}, \mathrm{k}_{\mathrm{n}}\right)}$ : Indicador de tráfico de carga que indica la carga transportada por cada unidad de distancia recorrida por el vehículo i:

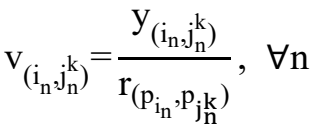

Definición de las variables de decisión $\left(\mathbf{X}_{\mathbf{n}}\right)$ : conjuntos de variables de $\mathfrak{R}^{m \times K}$ dimensiones que identifican las soluciones factibles del problema en cada etapa, de acuerdo a las variables de estado y las restricciones formuladas.

Cada variable perteneciente a un conjunto solución sería una variable binaria $x_{\left(i_{n}, j_{n}^{k}\right)}$ que puede tomar valores 0 y 1 indicando si el vehículo $i_{n}$ transporta mercancías o no al destino $j_{n}^{k}$ en la etapa $n$.

\subsection{Procedimiento para la formulación y resolución del modelo matemático.}

El modelo matemático, conforme al método heurístico de descomposición aplicado, resuelve cada etapa como un subproblema de optimización, siguiendo una serie de pasos secuenciales, con un procedimiento "en cascada" donde los resultados de cada etapa sirven de información relevante para la siguiente y donde las soluciones interrelacionadas de todas las Etapas representan la solución del problema global. El procedimiento a seguir es el siguiente y se sintetiza en la Figura 1.

A continuación, se explica cada etapa y paso del procedimiento y modelo matemático que se propone.

\section{Etapa $(\mathbf{n}=1)$}

Representa el primer viaje de toda la flota de vehículos en forma simultánea.

Paso 1. Se determinan las dimensiones y se calculan las variables de estado:

\section{Para los vehículos:}

$$
\begin{aligned}
& \mathrm{I}_{\mathrm{n}}=\mathrm{I}, \quad \forall \mathrm{n} \\
& \mathrm{i}_{\mathrm{n}}=\mathrm{i}, \quad \forall \mathrm{n} \\
& \mathrm{c}_{\mathrm{i}_{\mathrm{n}=1}}=\mathrm{C}_{\mathrm{i}}, \forall \mathrm{i} \\
& \mathrm{p}_{\mathrm{i}_{\mathrm{n}=1}}=0 \quad / 0=\text { Origen; }
\end{aligned}
$$


Figura 1. Procedimiento y modelo matemático para la planificación de ruteo vehicular.

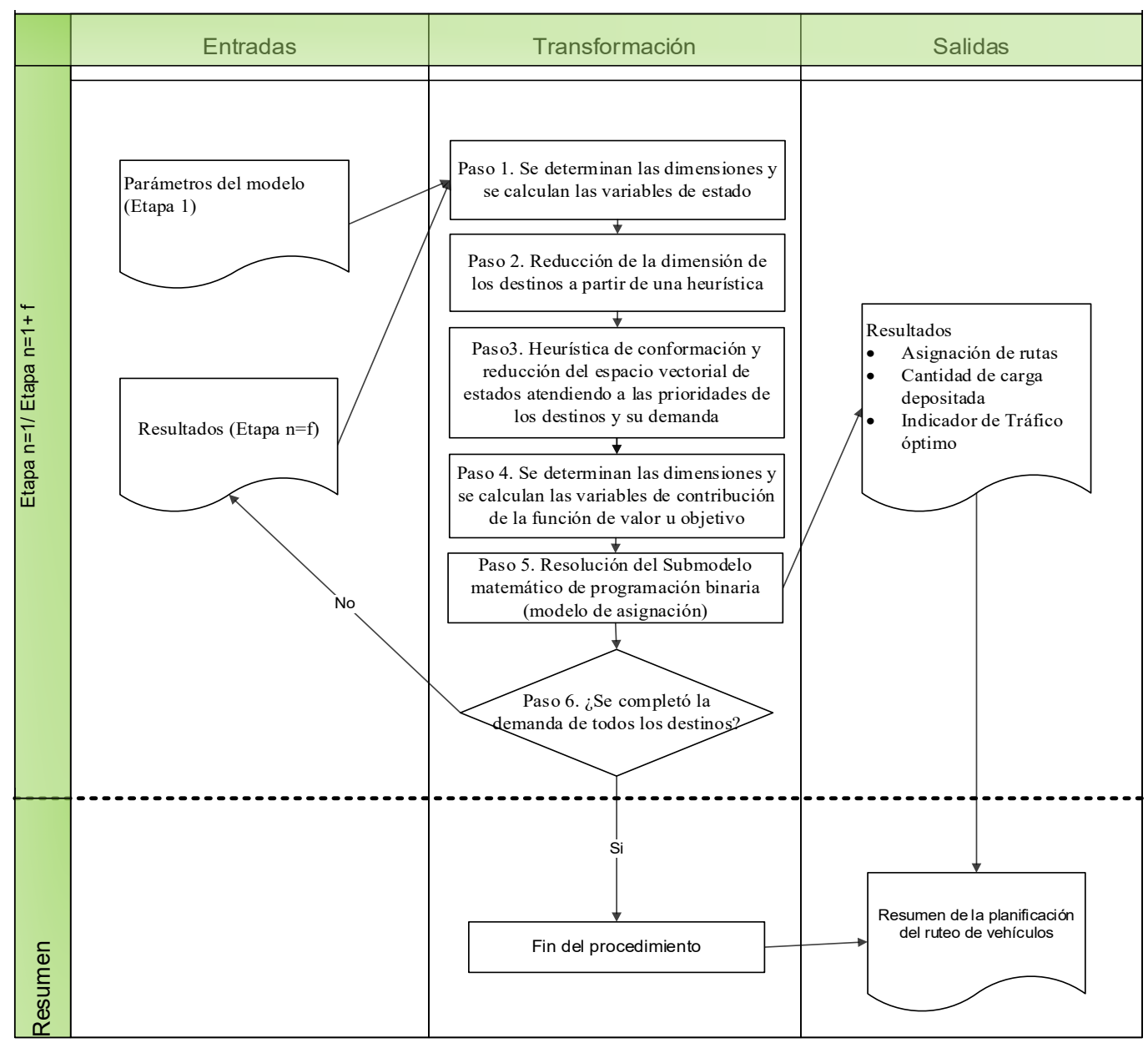

Fuente: Elaboración propia.

Como primer paso se definir las variables de estado del modelo en esta primera etapa, resultando en que el conjunto de vehículos a analizar es el total de vehículos con que se cuentan según se muestran en las ecuaciones (1) y (2). La ecuación (3) describe que la carga con que cuenta cada vehículo en esta primera etapa es la carga total al cargar en el origen o depósito. Por su parte la ecuación (4) denota que cada vehículo en esta etapa se encuentra físicamente en el origen o depósito.

\section{Para los destinos:}

$$
\begin{aligned}
& \mathrm{J}_{\mathrm{n}=1}=\mathrm{J}, \\
& \mathrm{j}_{\mathrm{n}}=\mathrm{j}, \\
& \mathrm{t}_{\mathrm{j}_{\mathrm{n}}=1}=\mathrm{T}_{\mathrm{j}}, \forall \mathrm{j} \\
& \mathrm{w}_{\mathrm{j}_{\mathrm{n}}}=\frac{\mathrm{t}_{\mathrm{n}}}{\mathrm{E}_{\mathrm{j}}}, \quad \forall \mathrm{n}
\end{aligned}
$$




$$
\begin{aligned}
& \mathrm{a}_{\mathrm{j}_{\mathrm{n}}}=\left\{\begin{array}{c}
1 \quad \text { para } \quad \mathrm{w}_{\mathrm{j}_{\mathrm{n}}}=0 \\
2 \quad \text { para } 0<\mathrm{w}_{\mathrm{j}_{\mathrm{n}}} \leq 1 \\
\text { hasta } \mathrm{b}+1 \quad \text { para } b-1<\mathrm{w}_{\mathrm{j}_{\mathrm{n}}}<\mathrm{b}
\end{array} \quad \forall \mathrm{n}\right. \\
& \mathrm{d}_{\mathrm{j}_{\mathrm{n}}}=\mathrm{E}_{\mathrm{j}} \times \mathrm{b}-\mathrm{t}_{\mathrm{j}_{\mathrm{n}}}, \quad \forall \mathrm{n}
\end{aligned}
$$

Por otra parte, las ecuaciones (5) y (6) señalan que en esta primera etapa, en un primer momento el conjunto de destinos a analizar es el total de destinos con que se cuenta. La ecuación (7) define que el inventario de mercancías de cada destino en esta etapa es igual al inventario inicial. La ecuación (8) calcula el tiempo de cobertura para esta etapa para cada destino de acuerdo al cociente entre el inventario de mercancías y consumo de mercancías promedio. La ecuación (9) por su parte muestra cómo se define el orden de prioridad en la atención de la demanda de cada destino de acuerdo a su nivel de cobertura calculado, hasta el nivel de cobertura meta definido como b. Por último, en la ecuación (10) se calcula la demanda para el transporte de mercancías de cada punto, de acuerdo a su consumo promedio y el nivel de cobertura meta planificado, descontando el nivel de inventario existente.

\section{Paso 2. Reducción de la dimensión de los destinos a partir de una heurística.}

Se aplica un método heurístico de reducción de la dimensión de los destinos a partir del criterio que solo es necesario analizar aquellos destinos cuyo nivel de inventario no garantice el tiempo de cobertura meta planificado. En el Algoritmo 1, se presenta la heurística propuesta en seudo-código.

\section{Algoritmo 1. Reducción de la dimensión de los destinos.}

//Se realiza para cada destino $\mathrm{j}_{\mathrm{n}}$

Consultar $\mathrm{w}_{\mathrm{j}_{\mathrm{n}}}$

Consultar b

$$
\text { Si } \mathrm{b}>\mathrm{w}_{\mathrm{j}_{\mathrm{n}}} \text { Entonces }
$$

// Implica que el inventario del destino j no garantiza el nivel de cobertura planificado y debe ser contemplado en el análisis

$$
\mathrm{j}_{\mathrm{n}} \in \mathrm{J}_{\mathrm{n}}
$$

$$
\text { SiNo } \mathrm{j}_{\mathrm{n}} \notin \mathrm{J}_{\mathrm{n}}, \mathrm{j}_{\mathrm{n}}=\phi
$$

//Implica que el inventario del destino $\mathrm{j}$ es igual o superior al nivel de cobertura planificado y por tanto sale del análisis.

\section{FinSi}

FinAlgoritmo

\section{Paso 3. Heurística de conformación y reducción del espacio vectorial de estado atendiendo a las prioridades de los destinos y su demanda.}

La heurística propuesta trata de replicar la forma en que se programa el ruteo actualmente. Para acotar el espacio vectorial de búsqueda del estado en cada etapa se ordenan los destinos por el nivel de prioridad previamente establecido como primer criterio y el valor de su demanda como segundo. Se 
define un subconjunto de la dimensión de los destinos de manera que se escogen los $m$ primeros destinos si la cantidad de destinos con demanda de mercancías sin atender es mayor que $m$, siendo $m$ igual al número de vehículos disponibles cuya ruta se quiere programar, o el número total de destinos si la cantidad de destinos con demanda de mercancías sin atender es menor o igual que $m$. Esto se realiza bajo el supuesto que un vehículo disponible para cargar mercancías solo puede ser asignado en una visita a un destino y a cada destino en cada vista solo se le debe asignar un vehículo.

Cada destino escogido se le denota como $\mathrm{j}_{\mathrm{n}}^{\mathrm{k}}, \mathrm{j}_{\mathrm{n}} \in \mathrm{J}_{\mathrm{n}}^{\mathrm{k}} / \mathrm{j}_{\mathrm{n}}=\mathrm{j}_{\mathrm{n}}^{\mathrm{k}}, \mathrm{k}=(1,2, \ldots, \mathrm{K})$ para los destinos escogidos por la heurística aplicada $\mathrm{y} \mathrm{j}_{\mathrm{n}} \notin \mathrm{J}_{\mathrm{n}}^{\mathrm{k}} / \mathrm{j}_{\mathrm{n}}^{\mathrm{k}}=\emptyset$ para los no escogidos. Luego se conforma un conjunto $\mathrm{X}_{\mathrm{n}}$ que estaría conformado por las variables binarias $\mathrm{x}_{\left(\mathrm{i}_{\mathrm{n}}, \mathrm{j}_{\mathrm{n}}^{\mathrm{k}}\right)}$, resultado de las combinaciones posibles entre los vehículos $i_{n} y$ los destinos escogidos $j_{n}^{k}$.

Paso 4. Se determinan las dimensiones y se calculan las variables de contribución de la función de valor u objetivo.

$$
\left.\mathrm{r}_{\left(\mathrm{p}_{\mathrm{i}_{\mathrm{n}=1}, \mathrm{p}_{\mathrm{n}=1}}\right)}=\mathrm{R}_{\left(\mathrm{p}_{\mathrm{i}_{\mathrm{n}}}, \mathrm{p}_{\mathrm{j}} \mathrm{k}\right.}\right)
$$

La ecuación (11) define que la distancia a recorrer por cada vehículo, como parte del modelo, depende de la posición física inicial del mismo y la posición física de los destinos que se contemplan en el análisis.

Para el cálculo de $\mathrm{y}_{\left(\mathrm{i}_{\mathrm{n}}, \mathrm{j}_{\mathrm{n}}^{\mathrm{k}}\right)}$ se sigue el Algoritmo 2 representado en seudo-código.

\section{Algoritmo 2. Determinar el volumen de mercancías a transportar.}

//Se realiza por cada combinación de vehículo i y destino $\mathrm{j}_{\mathrm{n}}^{\mathrm{k}}$

$$
\text { Si } \mathrm{c}_{\mathrm{i}_{\mathrm{n}}}>\mathrm{d}_{\mathrm{j}_{\mathrm{n}}} \text { : }
$$

Entonces $\mathrm{y}_{\left(\mathrm{i}_{\mathrm{n}}, \mathrm{j}_{\mathrm{n}}^{\mathrm{k}}\right)}=\mathrm{d}_{\mathrm{j}_{\mathrm{n}}^{\mathrm{k}}} / /$ Implica que la carga de mercancías en la etapa $\mathrm{n}$ del vehículo $\mathrm{i}$ es superior a la demanda del destino consultado por tanto solo se entrega el valor demandado.

SiNo $\mathrm{y}_{\left(\mathrm{i}_{\mathrm{n}}, \mathrm{j}_{\mathrm{n}}^{\mathrm{k}}\right)}=\mathrm{c}_{\mathrm{i}_{\mathrm{n}}} / /$ Implica que la carga de mercancías en la etapa $\mathrm{n}$ del vehículo $\mathrm{i}$ es inferior o igual a la demanda del destino consultado por tanto se entrega toda la carga.

\section{FinSi}

FinAlgoritmo

\section{Paso 5. Resolución del subproblema en la Etapa 1.}

Se formula un submodelo de optimización de Programación binaria, a partir de las variables relevantes ya determinadas sus dimensiones y calculados sus valores. Se busca la asignación óptima de vehículos disponibles a destinos escogidos por orden de prioridad, tomando como criterio la maximización del indicador tráfico de carga.

$$
\text { FO. Max. } \sum_{\mathrm{i}_{\mathrm{n}}=1}^{\mathrm{m}} \sum_{\mathrm{j}_{\mathrm{n}}^{\mathrm{k}}=1}^{\mathrm{k}=\mathrm{K}} \mathrm{v}_{\left(\mathrm{i}_{\mathrm{n}}, \mathrm{j}_{\mathrm{n}}^{\mathrm{k}}\right)} \times \mathrm{x}_{\left(\mathrm{i}_{\mathrm{n}}, \mathrm{j}_{\mathrm{n}}\right)} \mathrm{k}_{\mathrm{n}}
$$


sujeto a: $\sum_{\mathrm{i}_{\mathrm{n}}=1}^{\mathrm{m}} \mathrm{x}_{\left(\mathrm{i}_{\mathrm{n}}, \mathrm{j}_{\mathrm{n}}^{\mathrm{k}}\right)}=1$, solo se puede asignar un vehículo a un destino en la etapa $\mathrm{n}$

$$
\sum_{\mathrm{j}_{\mathrm{n}}^{\mathrm{k}=1}}^{\mathrm{k}=\mathrm{K}} \mathrm{x}_{\left(\mathrm{i}_{\mathrm{n}}, \mathrm{j}_{\mathrm{n}}^{\mathrm{k}}\right)}=1 \text {, solo se puede asignar a un destino, un vehículo en la etapa } \mathrm{n}
$$

$\mathrm{x}_{\left(\mathrm{i}_{\mathrm{n}}, \mathrm{k}_{\mathrm{n}}^{\mathrm{k}}\right)}\left\{\begin{array}{l}0, \text { si el vehículo i no transporta mercancías al destino } \mathrm{j} \text { en la etapa } \mathrm{n} \\ 1 \text {, si el vehículo i sí transporta mercancías al destino } \mathrm{j} \text { en la etapa } \mathrm{n}\end{array}\right.$

La función objetivo, expresión (12), relaciona las variables binarias con la cantidad de la mercancía a descargar en un destino de manera directamente proporcional, e inversamente proporcional con la distancia a la que se encuentra del origen, lo que se traduce en que se busca maximizar el tráfico de carga que se genera con el ruteo. La ausencia de información relevante para aplicar criterios económicamente más fiables como los costos de transporte, tiene una alternativa en el uso del indicador tráfico de carga frente a no presentar ninguna.

\section{Paso 6. Algoritmo de transición entre Etapas.}

El número de etapas está determinado por el número de destinos con un nivel de inventario por debajo del nivel de cobertura planificado en cada etapa representado por el conjunto $J_{n}$, de modo que el procedimiento acaba en la Etapa $n$ tal que, $\mathrm{J}_{\mathrm{n}}=\emptyset$, o conjunto vacío, lo que representaría que todos los destinos presentan un nivel de cobertura de su inventario igual o por encima del planificado.

\section{Etapa $(n=1+f / f=(1,2, \ldots, N-1)$.}

Representa todas las etapas que van a continuación de la primera etapa, en orden cronológico y consecutivo, y donde los resultados de cada etapa precedente constituyen parámetros en el submodelo representado por la etapa posterior, cumpliéndose que:

\section{Paso 1. Se determinan las dimensiones y se calculan las variables de estado:}

\section{Para los vehículos:}

Se calculan las ecuaciones (1) y (2) de acuerdo a la nueva etapa que se analiza.

Para el cálculo de $\mathrm{c}_{\mathrm{i}_{\mathrm{n}=1+\mathrm{f}}}$ se sigue el Algoritmo 3 representado en seudo-código. Este algoritmo implica que la carga de mercancías con que cuenta cada vehículo en esta etapa depende de la distribución planificada en la etapa anterior conforme a los resultados obtenidos.

\section{Algoritmo 3. Determinar el volumen de carga de cada vehículo en la Etapa $(1+f)$.}

//Se realiza por cada vehículo i y para cada destino $\mathrm{j}_{\mathrm{n}}^{\mathrm{k}}$ en la etapa anterior

Consultar $\mathrm{x}_{\left(\mathrm{i}_{\mathrm{n}=\mathrm{f}}, \mathrm{n}_{\mathrm{n}=\mathrm{f}}\right)} / /$ denota si al vehículo i le fue asignado o no transportar mercancías al destino $\mathrm{j}$ en la etapa anterior

Si $\mathrm{x}_{\left(\mathrm{i}_{\mathrm{n}=\mathrm{f}, \mathrm{j}=\mathrm{f}}^{\mathrm{k}}\right)}=1$ :

Entonces

Consultar $\mathrm{y}_{\left(\mathrm{i}_{\mathrm{n}=\mathrm{f}} ; \mathrm{j}_{\mathrm{n}=\mathrm{f}}^{\mathrm{k}}\right)}$

Consultar $\mathrm{c}_{\mathrm{i}_{\mathrm{n}=\mathrm{f}}}$ 


$$
\text { Si } \mathrm{c}_{\mathrm{i}_{\mathrm{n}=\mathrm{f}}}>\mathrm{y}_{\left(\mathrm{i}_{\mathrm{n}=\mathrm{f}}, \mathrm{j}_{\mathrm{n}=\mathrm{f}}^{\mathrm{k}}\right)} \text { : }
$$

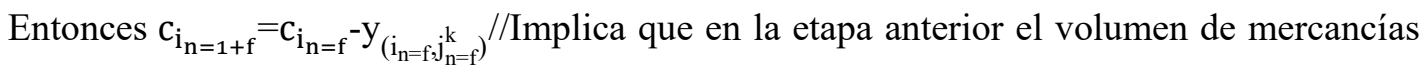
descargado por el vehículo i era menor que el transportado, por tanto mantiene la diferencia.

SiNo $\mathrm{c}_{\mathrm{i}_{\mathrm{n}=1+\mathrm{f}}}=\mathrm{C}_{\mathrm{i}} / /$ Implica que en la etapa anterior el vehículo i descargó toda su carga y por tanto vuelve al origen depósito a cargar.

FinSi

SiNo $\mathrm{c}_{\mathrm{i}_{\mathrm{n}=1+\mathrm{f}}}=\mathrm{Nulo}$

FinSi

FinAlgoritmo

$\mathrm{p}_{\mathrm{i}_{\mathrm{n}=1+\mathrm{f}}}$ : Para su determinación se utiliza el Algoritmo 4 mostrado en seudo-código. Este algoritmo define que la posición física de cada vehículo en esta etapa depende de la distribución planificada en la etapa anterior conforme a los resultados obtenidos.

\section{Algoritmo 4. Determinar la posición física de cada vehículo en la Etapa (1+f).}

//Se realiza por cada vehículo i en esta etapa y para cada destino $\mathrm{j}_{\mathrm{n}}^{\mathrm{k}}$ en la etapa anterior

Consultar $\mathrm{x}_{\left(\mathrm{i}_{\mathrm{n}=\mathrm{f}, \mathrm{j}=\mathrm{f}}, \mathrm{k}\right.} / /$ denota si al vehículo i le fue asignado o no transportar mercancías al destino $\mathrm{j}$ en la etapa anterior

Si $\mathrm{x}_{\left(\mathrm{i}_{\mathrm{n}=\mathrm{f}, \mathrm{j}}, \mathrm{k}, \mathrm{f}\right)}=1$ :

Entonces $\mathrm{p}_{\mathrm{i}_{\mathrm{n}=1+\mathrm{f}}}=\mathrm{p}_{\mathrm{j}_{\mathrm{n}=\mathrm{f}}} / /$ Implica que el vehículo i se encuentra al inicio de la etapa actual en la posición del destino j que le fue asignado transportar mercancías en la etapa anterior

$$
\text { SiNo } \mathrm{p}_{\mathrm{i}_{\mathrm{n}=1+\mathrm{f}}}=\text { Nulo }
$$

FinSi

FinAlgoritmo

\section{Para los destinos:}

$$
\mathrm{J}_{\mathrm{n}=1+\mathrm{f}}=\mathrm{J}_{\mathrm{n}=\mathrm{f}}
$$

La ecuación (15) define que en un primero momento el conjunto de destinos analizados en la etapa actual es igual al conjunto de la etapa anterior.

Para el cálculo de $\mathrm{t}_{\mathrm{j}_{\mathrm{n} 1+\mathrm{f}}}$ se sigue el Algoritmo 5 representado en seudo-código. Este algoritmo contempla los resultados obtenidos de la distribución planificada en la etapa anterior para calcular el inventario de mercancías actualizado de cada destino dentro del análisis. 
//Se realiza por cada destino j en esta etapa y para cada vehículo i en la etapa anterior

$$
\text { Si } \mathrm{j}_{\mathrm{n}=\mathrm{f}}=\mathrm{j}_{\mathrm{n}=\mathrm{f}}^{\mathrm{k}} \text { : }
$$

Entonces//Implica que el destino j fue seleccionado por la heurística de reducción en la etapa anterior y por tanto se le asignó el transporte de mercancías

$$
\text { Si } x_{\left(\mathrm{i}_{\mathrm{n}=\mathrm{f}, \mathrm{j}}^{\mathrm{j}=\mathrm{f}} \mathrm{k}\right)}^{\mathrm{k}}=1 \text { : }
$$

Entonces $\mathrm{t}_{\mathrm{j}_{\mathrm{n}=1+\mathrm{f}}}=\mathrm{t}_{\mathrm{j}_{\mathrm{n}=\mathrm{f}}}+\mathrm{y}_{\left(\mathrm{i}_{\mathrm{n}=\mathrm{f} ;} ; \mathrm{j}_{\mathrm{n}=\mathrm{f}} \mathrm{k}\right)} / /$ Implica que el inventario del destino $\mathrm{j}$ en la etapa actual es igual al inventario de la etapa anterior más el volumen de mercancías transportado por el vehículo i en la etapa anterior

$$
\text { SiNo } \mathrm{t}_{\mathrm{j}_{\mathrm{n}=1+\mathrm{f}}}=\text { Nulo }
$$

FinSi

SiNo $\mathrm{t}_{\mathrm{j}_{\mathrm{n}=1+\mathrm{f}}}=\mathrm{t}_{\mathrm{j}_{\mathrm{n}=\mathrm{f}}} / /$ Implica que el destino $\mathrm{j}$ mantiene el nivel de inventario de la etapa previa FinSi

FinAlgoritmo

Se calculan las ecuaciones (3), (4), (5) y (6).

Paso 2. Reducción de la dimensión de los destinos a partir de una heurística (Í́dem a la Etapa 1).

Paso 3. Heurística de conformación y reducción del espacio vectorial de estados atendiendo a las prioridades de los destinos y su demanda (Ídem a la Etapa 1).

Paso 4. Se determinan las dimensiones y se calculan las variables de contribución de la función de valor u objetivo. 1.

Para el cálculo de $\mathrm{y}_{\left(\mathrm{i}_{\mathrm{n}=1+\mathrm{F}}, \mathrm{j}_{\mathrm{n}=1+\mathrm{f}}^{\mathrm{k}}\right)}$ se sigue el Algoritmo 2 representado en seudo-código en la Etapa

Para el cálculo de $\mathrm{r}_{\left(\mathrm{p}_{\mathrm{i}_{\mathrm{n}}+\mathrm{f}_{\mathrm{f}}} \mathrm{p}_{\mathrm{j}_{\mathrm{n}}^{\mathrm{k}} \mathrm{k}+\mathrm{f}}\right)}$ se sigue el Algoritmo 6 representado en seudo-código. Con este algoritmo se obtiene la distancia a recorrer por cada vehículo en esta etapa, dependiendo de la distribución planificada en la etapa anterior conforme a los resultados obtenidos y, por tanto, de la posición física inicial y final de cada vehículo en esta etapa. 


\section{Algoritmo 6. Determinar la distancia a recorrer por cada vehículo en la Etapa (1+f).}

//Se realiza por cada vehículo i y para cada destino $\mathrm{j}_{\mathrm{n}}^{\mathrm{k}}$ en la etapa anterior

Consultar $\mathrm{x}_{\left(\mathrm{i}_{\mathrm{n}=\mathrm{f}}, \mathrm{j}_{\mathrm{n}=\mathrm{f}}\right)} / /$ denota si al vehículo i le fue asignado o no transportar mercancías al destino $\mathrm{j}$ en la etapa anterior

Si $x_{\left(i_{n=f}, j_{n=f}^{k}\right)}=1$ :

Entonces

Si $\mathrm{c}_{\mathrm{i}_{\mathrm{n}=\mathrm{f}}}>\mathrm{y}_{\left(\mathrm{i}_{\mathrm{n}=\mathrm{f}}, \mathrm{j}_{\mathrm{n}=\mathrm{f}}^{\mathrm{k}}\right)}$ :

Entonces $\left.\mathrm{r}_{\left(\mathrm{p}_{\mathrm{i}_{\mathrm{n}}+\mathrm{f}+\mathrm{p}} \mathrm{p}_{\mathrm{n}=1+\mathrm{f}}\right)}=\mathrm{R}_{\left(\mathrm{p}_{\mathrm{i}=1+\mathrm{f}}, \mathrm{p}_{\mathrm{n}}^{\mathrm{k}} \mathrm{k}=1+\mathrm{f}\right.}\right) / /$ Implica que en la etapa anterior el volumen de mercancías descargado por el vehículo i era menor que el transportado, por tanto no tiene que volver al origen a cargar.

SiNo $\left.\mathrm{r}_{\left(\mathrm{p}_{\mathrm{i}_{\mathrm{n}}+\mathrm{f}} \mathrm{f}_{\mathrm{j}_{\mathrm{n}=1+\mathrm{f}}}\right)}=\mathrm{R}_{\left(\mathrm{p}_{\mathrm{i}_{\mathrm{n}}=1+\mathrm{f}}, 0\right)}+\mathrm{R}_{\left(0, \mathrm{p}_{\mathrm{j}=1+\mathrm{f}}^{\mathrm{k}}\right.}\right) / /$ Implica que en la etapa anterior el vehículo $\mathrm{i}$ descargó toda su carga y por tanto vuelve al origen a cargar, recorriendo esa distancia antes de visitar el próximo destino.

FinSi

SiNo $\left.\mathrm{r}_{\left(\mathrm{p}_{\mathrm{i}_{\mathrm{n}=1+\mathrm{f}}}, \mathrm{p}_{\mathrm{j}} \mathrm{k}=1+\mathrm{f}\right.}\right)$ Nulo

FinSi

FinAlgoritmo

Paso 5. Resolución del subproblema en la Etapa (Ídem a la Etapa 1).

Paso 6. Algoritmo de transición entre Etapas (Ídem a la Etapa 1).

\section{Aplicación del modelo en un caso de estudio.}

El modelo propuesto ha sido evaluado con datos relativos a un caso de estudio a partir de una situación real en la planificación de distribución en la empresa del problema que suscita la investigación. Asimismo, se realiza una evaluación de los resultados obtenidos por el modelo que se propone a partir de los datos asociados al caso de estudio y se compara con los resultados obtenidos por la planificación empírica real realizada.

\subsection{Implementación y resolución.}

Los modelos propuestos han sido implementados con el lenguaje de programación Python, en su versión 3.7.3, que es un lenguaje de alto nivel adecuado para el código científico y de ingeniería que a menudo es lo suficientemente rápido como para ser inmediatamente útil, pero también lo suficientemente flexible como para ser acelerado con extensiones adicionales (Oliphant, 2007). Se utilizó el módulo Pyomo, versión 5.6.8, que es una extensión que proporciona una capacidad dentro de Python comúnmente asociada con lenguajes de modelado algebraico como AMPL y GAMS (Hart, 2009). En 
conjunto con Pyomo se usó el solver de optimización CBC. Se empleó una PC Core i3-4130 CPU @3.40 GHz, $3400 \mathrm{Mhz}$, con $4 \mathrm{~GB}$ de Memoria RAM en un tiempo relativamente corto.

Conforme al problema definido, el modelo ha sido ejecutado para un periodo de planificación de 1 día, con 13 vehículos aptos para el transporte y 70 puntos de ventas o destinos. La Tabla 2, recogida en el Anexo I, muestra los datos básicos asociados a los vehículos, la marca y la capacidad total en unidades. Por otro lado, la Tabla 3 (Anexo II) recoge los datos de los puntos de venta que constituyen los destinos, su inventario inicial consumo promedio y nivel de cobertura planificado.

\subsection{Comparación de resultados.}

Los resultados más relevantes de la resolución del modelo y su comparación con los resultados reales de la programación operativa realizada por la empresa para esta situación puntual se muestran en la Tabla 1.

Tabla 1. Comparación entre resultados reales y los obtenidos con el modelo matemático.

\begin{tabular}{ccc}
\hline Indicador & Real & Modelo \\
\hline Cantidad de destinos con menos de 3 días de cobertura al inicio del día (unidad) & 14 & 14 \\
Cantidad de destinos con menos de $3 \begin{array}{c}\text { días de cobertura en sus inventarios al final del día } \\
\text { (unidad) }\end{array}$ & 5 & 0 \\
Carga transportada (toneladas) & 85,65 & 85,00 \\
Distancia recorrida por los vehículos (kilómetros) & 450,80 & 274,20 \\
Tráfico de carga (t/km) & 0,19 & 0,31 \\
Fuente: Elaboración propia. & &
\end{tabular}

El modelo matemático de ruteo vehicular y manejo de inventario diseñado resulta viable para la entidad objeto de investigación y ha permitido diseñar una programación ruteo para un caso de estudio específico basado en la realidad de la gestión operativa de la misma. Este modelo responde en su diseño a un método heurístico de descomposición, que se combina con otros dos métodos heurísticos de reducción. Este tipo de métodos algunos autores los denominan como algoritmos a la medida, no siendo aprovechables para un problema diferente al que fue diseñado (Piqueras \& Folgado, 2002). Con este modelo se construye un óptimo local del problema en cada etapa en la que se subdivide a partir del uso de la programación lineal binaria. Sin embargo, la solución encontrada no garantiza el óptimo global, pues no sigue el principio de optimalidad típico de la programación dinámica que plantea que una política óptima para las etapas restantes es independiente de la política adoptada en etapas anteriores (Delgado et al., 2015). No obstante, la técnica empleada evita explorar todas las secuencias posibles por medio de la resolución de subproblemas y almacenamiento en una tabla de las soluciones óptimas de esos subproblemas para facilitar la solución del problema más grande. Se justifica este proceder basado en el costo en tiempo y otros recursos computacionales que implicaría desarrollar un procedimiento para hallar una solución óptima que, por otra parte, dado el caso al que nos referimos, no representará un beneficio importante con respecto a otra que sea simplemente satisfactoria.

La utilización del modelo formulado como procedimiento operativo para el ruteo vehicular en la distribución de materias primas en la empresa tiene el potencial de contribuir a una utilización más racional de los vehículos disponible. En el caso de estudio se logra completar la cobertura planificada 
del inventario con respecto a la demanda de todos los destinos, en contraposición en la gestión real quedaron por debajo del nivel de cobertura 5 destinos. Así mismo, el valor del indicador tráfico de carga total, para el caso del ruteo obtenido como parte del modelo, es de 0,31 toneladas por kilómetro recorrido, superior al valor de 0,19 que se obtuvo con la planificación real. Todo esto amparado en un potencial ahorro de kilómetros recorridos por los vehículos de 176,6 con un aumento en el volumen de carga transportada de 0,65 toneladas. Aun cuando hay que tomar con cautela una comparación que no cuenta con un tamaño de muestra que garantice fiabilidad, y basada en un caso hipotético, representa una posibilidad de mejora latente.

\section{Conclusiones.}

En este trabajo se plantea un problema de ruteo vehicular asociado al manejo de inventario, o Inventory Routing Problem (IRP) en un caso particular dado en una entidad comercializadora de gas licuado de la región oriental de Cuba. La particularidad del problema planteado, y no recogido anteriormente en la literatura revisada, se debe a la utilización como objetivo a resolver en el problema formulado del indicador tráfico de carga.

La contribución de esta investigación es la formulación de dicho problema, así como una propuesta de solución mediante un modelo matemático heurístico integrado. El modelo junto al procedimiento que se sigue para su resolución, responde a las características del proceso de distribución física en la entidad objeto de investigación. Éste fue evaluado en un caso de estudio basado en una situación problemática real y puntual de la empresa, proporcionando una programación de ruteo vehicular con un resultado satisfactorio en comparación con los resultados del modo de proceder actual.

En cuanto a las líneas futuras de actuación, a corto plazo la empresa debe estudiar la viabilidad de introducir el procedimiento como parte de la planificación operativa de la distribución de mercancías. Sería interesante el estudio de la aplicación del modelo y procedimiento propuesto bajo la óptica de un Sistema de apoyo a la toma de decisiones, o Decision Support Systems (DSS); aprovechando la estrecha relación existente y en incremento entre los modelos matemáticos, la gestión empresarial y las Tecnologías de la Información y las Comunicaciones (TIC's).

\section{Referencias}

Andersson, H., Hoff, A., Christiansen, M., Hasle, G., \& Løkketangen, A. (2010). Industrial aspects and literature survey: Combined inventory management and routing. Computers \& Operations Research, 37(9), 1515-1536.

Archetti, C., Coelho, L.C., \& Speranza, M.G. (2019). An exact algorithm for the inventory routing problem with logistic ratio. Transportation Research Part E: Logistics and Transportation Review, 131, 96-107.

Archetti, C., Desaulniers, G., \& Speranza, M.G. (2017). Minimizing the logistic ratio in the inventory routing problem. EURO Journal on Transportation and Logistics, 6(4), 289-306.

Braekers, K., Ramaekers, K., \& Van Nieuwenhuyse, I. (2016). The vehicle routing problem: State of the art classification and review. Computers \& Industrial Engineering, 99, 300-313.

Campbell, A., Clarke, L., Kleywegt, A., \& Savelsbergh, M. (1998). The inventory routing problem. In Fleet management and logistics (pp. 95-113). Boston, MA: Springer.

Campbell, A.M., \& Savelsbergh, M.W. (2004). A decomposition approach for the inventory-routing problem. Transportation science, 38(4), 488-502. 
Cattaruzza, D., Absi, N., Feillet, D., \& González-Feliu, J. (2017). Vehicle routing problems for city logistics. EURO Journal on Transportation and Logistics, 6(1), 51-79.

Coelho, L.C., Cordeau, J.F., \& Laporte, G. (2014). Thirty years of inventory routing. Transportation Science, 48(1), 1-19.

Delgado, J.A.C., Avalos, L.C.M., Delgado, E.R., \& Puycán, L.A.L. (2015). Optimización de programas matemáticos con programación dinámica. Ciencia \& Desarrollo, 19, 77-83.

Eksioglu, B., Vural, A.V., \& Reisman, A. (2009). The vehicle routing problem: A taxonomic review. Computers \& Industrial Engineering, 57(4), 1472-1483.

Hart, W.E. (2009). Python optimization modeling objects (Pyomo). In Operations Research and CyberInfrastructure (pp. 3-19). Boston, MA: Springer.

Lao, Y.O., Vega, L.O., Marrero, F., \& Pérez, M.C. (2017). Procedimiento para modelar recursos restrictivos en el sistema logístico de empresas comercializadoras. Ingeniería Industrial, 38(1), 43-55.

Li, K., Chen, B., Sivakumar, A.I., \& Wu, Y. (2014). An inventory-routing problem with the objective of travel time minimization. European Journal of Operational Research, 236(3), 936-945.

Oliphant, T.E. (2007). Python for scientific computing. Computing in Science \& Engineering, 9(3), 1020.

ONEI (2019). Anuario Estadístico de Cuba 2018. La Habana, Cuba, Edición 2019.

Palhares, R.A., \& Araújo, M.C.B. (2018, December). Vehicle Routing: Application of Travelling Salesman Problem in a Dairy. In 2018 IEEE International Conference on Industrial Engineering and Engineering Management (IEEM) (pp. 1421-1425). IEEE.

Pecin, D., Contardo, C., Desaulniers, G., \& Uchoa, E. (2017). New enhancements for the exact solution of the vehicle routing problem with time windows. INFORMS Journal on Computing, 29(3), 489-502.

Piqueras, V.Y., \& Folgado, J.R.M. (2002). Optimización heurística económica aplicada a las redes de transporte del tipo VRPTW. Valencia: Universidad Politécnica de Valencia.

Rahim, M.K.I.A., Iteng, R., \& Ahmad, M.A. (2017). A Deterministic Inventory Routing Model for the Single-period Problems with Finite Time Horizon. International Journal of Supply Chain Management, 6(2), 196-201.

Ritzinger, U., Puchinger, J., \& Hartl, R.F. (2016). A survey on dynamic and stochastic vehicle routing problems. International Journal of Production Research, 54(1), 215-231.

Soysal, M., Bloemhof-Ruwaard, J.M., Haijema, R., \& Van der Vorst, J.G. (2018). Modeling a green inventory routing problem for perishable products with horizontal collaboration. Computers \& Operations Research, 89, 168-182.

Uchoa, E., Pecin, D., Pessoa, A., Poggi, M., Vidal, T., \& Subramanian, A. (2017). New benchmark instances for the capacitated vehicle routing problem. European Journal of Operational Research, 257(3), 845-858. 
ANEXO I. Datos sobre los vehículos.

Tabla 2. Parámetros para la solución del modelo respecto a los vehículos.

\begin{tabular}{ccc}
\hline Id(i) & Marca & Capacidad en unidades (Ci) \\
\hline 1 & INTERNACIONAL & 460 \\
2 & INTERNACIONAL & 460 \\
3 & SINOTRUK & 364 \\
4 & SINOTRUK & 364 \\
5 & Hyundai & 342 \\
6 & KAMAZ & 310 \\
7 & Hyundai & 342 \\
8 & Hyundai & 342 \\
9 & Hyundai & 342 \\
10 & Hyundai & 342 \\
11 & Hyundai & 342 \\
12 & INTERNACIONAL & 460 \\
13 & INTERNACIONAL & 460 \\
& Fuente: Elaboración propia. &
\end{tabular}

ANEXO II. Datos sobre los Puntos de ventas o destinos.

Tabla 3. Parámetros para la solución del modelo respecto a los destinos.

\begin{tabular}{cccc}
\hline Id (j) & $\begin{array}{c}\text { Inventario inicial en } \\
\text { unidades (Tj) }\end{array}$ & $\begin{array}{c}\text { Consumo promedio en } \\
\text { unidades por día (Ej) }\end{array}$ & $\begin{array}{c}\text { Nivel de cobertura } \\
\text { meta en días (b) }\end{array}$ \\
\hline 1 & 135 & 29 & 3 \\
2 & 129 & 20 & 3 \\
3 & 146 & 21 & 3 \\
4 & 83 & 42 & 3 \\
5 & 130 & 28 & 3 \\
6 & 109 & 39 & 3 \\
7 & 146 & 46 & 3 \\
8 & 131 & 28 & 3 \\
9 & 127 & 34 & 3 \\
10 & 122 & 35 & 3 \\
11 & 38 & 64 & 3 \\
12 & 178 & 82 & 3 \\
13 & 84 & 100 & 3 \\
14 & 157 & 50 & 3 \\
15 & 250 & 68 & 3 \\
16 & 0 & 94 & 3 \\
17 & 199 & 75 &
\end{tabular}




\begin{tabular}{|c|c|c|c|}
\hline 18 & 229 & 75 & 3 \\
\hline 19 & 204 & 63 & 3 \\
\hline 20 & 199 & 84 & 3 \\
\hline 21 & 183 & 75 & 3 \\
\hline 22 & 181 & 58 & 3 \\
\hline 23 & 114 & 55 & 3 \\
\hline 24 & 98 & 30 & 3 \\
\hline 25 & 188 & 43 & 3 \\
\hline 26 & 181 & 84 & 3 \\
\hline 27 & 130 & 75 & 3 \\
\hline 28 & 0 & 37 & 3 \\
\hline 29 & 57 & 46 & 3 \\
\hline 30 & 198 & 64 & 3 \\
\hline 31 & 56 & 61 & 3 \\
\hline 32 & 195 & 73 & 3 \\
\hline 33 & 0 & 72 & 3 \\
\hline 34 & 181 & 73 & 3 \\
\hline 35 & 107 & 61 & 3 \\
\hline 36 & 139 & 45 & 3 \\
\hline 37 & 156 & 43 & 3 \\
\hline 38 & 123 & 78 & 3 \\
\hline 39 & 128 & 27 & 3 \\
\hline 40 & 65 & 72 & 3 \\
\hline 41 & 151 & 46 & 3 \\
\hline 42 & 52 & 83 & 3 \\
\hline 43 & 89 & 60 & 3 \\
\hline 44 & 83 & 76 & 3 \\
\hline 45 & 114 & 41 & 3 \\
\hline 46 & 157 & 20 & 3 \\
\hline 47 & 99 & 26 & 3 \\
\hline 48 & 93 & 22 & 3 \\
\hline 49 & 85 & 24 & 3 \\
\hline 50 & 82 & 35 & 3 \\
\hline 51 & 111 & 35 & 3 \\
\hline 52 & 67 & 37 & 3 \\
\hline 53 & 99 & 27 & 3 \\
\hline 54 & 90 & 71 & 3 \\
\hline 55 & 53 & 63 & 3 \\
\hline 56 & 4 & 105 & 3 \\
\hline 57 & 4 & 60 & 3 \\
\hline 58 & 212 & 105 & 3 \\
\hline 59 & 70 & 47 & 3 \\
\hline 60 & 94 & 59 & 3 \\
\hline 61 & 48 & 56 & 3 \\
\hline 62 & 103 & 32 & 3 \\
\hline 63 & 82 & 54 & 3 \\
\hline
\end{tabular}


64

65

66

67

68

69

70
154

81

146

13

14

9

5
48

55

38

115

4

3

31
3

3

3

3

3

3

3

Fuente: Elaboración propia. 